\title{
Review: Managing Very Challenging Behaviour
}

Managing Very Challenging Behaviour, by Louisa Leaman. New York, NY: Continuum International Publishing Group, 2005. Reviewed by Elizabeth Berger, MFA, Assistant Professor of Design, Zayed University, Dubai, UAE.

Louisa Leaman, author of Managing Very Challenging Behaviour, states that effective behavior management should be about supporting students so that they can manage their own behavior. Her perspective is from her professional experience with students in mainstream and special education settings in both primary and secondary education programs. While the content is about a younger group than university level students, often the emotional problems discussed are not different, and the solutions and techniques would be applicable to a higher education environment. The teaching behaviors, as well, are sound for all effective teachers. When you consider that new studies show that more advanced emotional development of brain functions develop in the later teen and young adult years the ideas presented by Leaman are critical to getting across information to students in an effective way. This book deals with issues I find to be very practical for higher education faculty.

Students in this age group are still developing in neural and emotional functions. Our role in effecting their growth can be so much more effective if we are aware of such issues and respond properly. While the ideas in this book are often about specific behaviors that can disrupt learning in the classroom, which may not be as applicable on the university level, the teaching techniques are very helpful and appropriate to college level students, as well as colleagues.

The book is written in a simple style for various levels of reader expertise in human behavior issues. It is organized into 11 sections that move from defining the issues, terms and behaviors and explaining the root causes of problem behaviors to elaborating on the management and actions to be taken in the classroom. Section 1 explores the underlying reasons for students' behaviors. Section 2 continues to break down the three root causes of most behavior problems: low self-esteem, egocentricity, and internalized anger. Section 3 builds on the backbone of management: assert clear boundaries, be consistent, use a sliding scale of intervention, emphasize choice, use "time-out", provide meaningful rewards and consequences, and finally the specific rewards and consequences.

In section 4 the author takes you through more behavior management skills of proactive, reactive and follow-up techniques. The next section, section 5 , takes you into very detailed ways of handling lowlevel disruptions with an emphasis on setting up a proper atmosphere, having a calm demeanor, moving toward the problem, working with the problem, rewarding improvements, and monitoring and sharing information. Section 6 discusses attention-seeking students and modeling behavior. There is an interesting discussion of the pros and cons of ignoring student behavior, and again it emphasizes rewards, setting specific expectations, and sharing information with colleagues. Section 7 focuses more on students with bad attitudes toward authority figures, and encourages the teacher to remain neutral and to turn negatives into positives.

At the beginning of section 8 the book goes into the next level of medium-level disruption, and teaching techniques such as awareness, monitoring behavior triggers, asserting boundaries, being repetitive and giving choices. An interesting point in this chapter is about accepting sincerity from a student and 
starting again with a clean slate. The last sections, 9 and 10, go further into medium and high level problems and emphasize giving space and time, and calling for back up when necessary from faculty.

Leaman discusses emotional and social behavior disorders (ESBD) and breaks them into three key areas: low self-esteem, egocentricity, and internalized anger. She elaborates on how these issues can mask very diverse behaviors in the classroom. She approaches solutions in a holistic way including the students accountability, the faculty, department, family and social structure. She encourages collaboration, awareness and taking a proactive stance in issues. The author uses the term "moving toward the problem."

Understanding the root cause of various classroom behaviors that can disrupt learning is also applicable to understanding various cultural differences in responding to emotional and social issues. In a multicultural environment this knowledge can be the difference between success and failure as a teacher.

Leaman points out that society often labels people the "lazy ones" or the other extreme the "show offs", when the root cause is often similar - low self-esteem. Labels can be very damaging to students and as teachers we have a responsibility to be aware of the depths of human behavior and to avoid labels or limits of a student's choices for his/her future. Teachers working in tandem within a well-run department should discuss and share insights into student behavior, styles of communication and possible solutions. This can greatly enhance a consistent curriculum and teaching atmosphere that can support student growth and academic experience.

The topic of fear came up in this book often; fears are a significant aspect of development in this age group - fears about inadequacy, status or acceptance are still major issues for university level students. Students fear being controlled or losing control, especially if they are from a violent or abusive home. According to Leaman, teachers that have strong fear-oriented, authoritative teaching styles will only worsen such behavior in this type of student. "For many students from troubling or emotionally unstable homes, school can offer a haven of fairly administered structure and emotional support. It is open to question, however, whether education can or should focus on emotional development per se. Perhaps the best lesson to be gleaned from our increased knowledge and understanding of emotional development, is that environments that are safe, fostering, and avoid potentially negative labels, will create the best climate for both social and cognitive growth" (Burns, 2006). Leaman suggests that our style of teaching, the consistency of our teamwork and the type of schools we create will not only foster growth in the ESBD student, but also help balance in the classroom.

The author discusses how faculty from western cultures (this may be true of other cultures as well), have a tendency to believe that winning through dominance is how things are done. If this were true of the cultural environment you are in, then a student from such a culture would not want to "lose face" with a teacher in a direct power confrontation. They may not want to admit weakness, or confront an issue, but deal with a problem through a more passive-aggressive means. This can manifest through overcompensating with a bravado that is not entirely genuine to his core personality, but reflects an unmet need or trauma. Teachers that are aware of behavioral core issues would be more equipped to empathically handle such a student according to the author. Again, awareness is the point of this book, to raise your awareness level and effectively create a classroom atmosphere that will deal with challenges in a positive way.

The backbone of behavior management according to Leaman is: assert clear boundaries, be consistent, use a sliding scale of intervention, emphasize choice for the student, use time-outs for breaks, provide meaningful rewards and consequences. 
These simple skills are often forgotten on the college level, yet at this developmental age, late-teens to early adult years, there are still environmental issues and behaviors a school and teacher can effect to critically guide development and therefore future success in a student. The author's methodology is to first look, then raise the voice slightly, and lastly to issue a warning that emphasizes the student's choice in a calm voice. A bullying teacher can gain control in the short run, but rob the student of his/her longterm behavioral growth.

The author states, "Be firm; Be fair; Be calm; Be clear and Be positive" a simple quote but a good mantra to remember when dealing with various ages, emotional levels of development in the classroom and among colleagues from various cultures.

The effective teacher has much more to do than convey information, but to prepare a young adult to be effective in his/her life and career. To do this task he/she must plan, prepare, provide and monitor the education of a large number of diverse students. Teamwork among faculty, especially in the more difficult situations where a student is failing to thrive, is imperative. Administrative leadership in monitoring and focusing goals and solutions will create a well-rounded experience for a wide range of students and faculty.

While this book is from the perspective of pre-tertiary education, it contains some very focused truths on human interaction and recommends pointed techniques for teachers. The book reaffirms the need for collaboration among faculty, modeling of behavior, and keeping your own emotions in check as you respond to students with their best interest in mind. From a faculty perspective I think this book is a valuable resource with simple steps and effective behaviors in dealing with students. The knowledge gained from reading this book would enhance effectiveness in the classroom. The clear delineation of steps guide you from the teacher's personal feelings and mental assessment of the behavioral situation to departmental support, administrative guidance and social support services. Leaman has written a very valuable resource for faculty at various teaching levels and institutions that deal with very realworld issues facing academia today.

\section{References}

Burns, M. (2006). Can Emotional Skills be Taught? Retrieved 28 June 2007 from

<http://www.brainconnection.com/topics/?main=col/burns00jul6>. 